Araştırma Makalesi / Research Article

Iğdır Üniversitesi Fen Bilimleri Enstitüsü Dergisi, 10(4): 2872-2880, 2020

Journal of the Institute of Science and Technology, 10(4): 2872-2880, 2020

ISSN: 2146-0574, eISSN: 2536-4618

\title{
Infinitesimal Paraholomorphically Projective Transformation On Cotangent Bundle With Riemannian Extension
}

\section{Lokman BİLEN $^{1 *}$}

ABSTRACT: The main purpose of the present paper is to study some properties of infinitesimal paraholomorphically projective transformation on $T^{*} M$ with respect to the Levi-Civita connection of the Riemannian extension $\left({ }^{R} \nabla\right)$ and adapted almost paracomplex structure $J$. Moreover, if $T^{*} M$ be admits a non-affine infinitesimal paraholomorphically projective transformation, than $M$ and $T^{*} M$ are locally flat.

Keywords: Paraholomorphically projective transformation, almost paracomplex structure, Riemannian extension, adapted frame.

${ }^{1}$ Lokman BİLEN (Orcid ID: 000000018240 5359), Igdir University, Faculty of Science and Letters, Department of Mathematics, Igdir, Turkey

*Corresponding Author: Lokman BİLEN, e-mail: lokman.bilen@igdir.edu.tr

Geliş tarihi / Received: 13-03-2020

Kabul tarihi / Accepted: 07-06-2020 


\section{INTRODUCTION}

Let $M$ be an $n$-dimensional manifold and $T^{*} M$ its cotangent bundle. Note that in the present paper everything will be always discussed in the $C^{\infty}$ - category, manifolds will be assumed to be connected and dimension $n>1$. And let $\pi$ the natural projection $T^{*} M \rightarrow M$. The local coordinates $\left(U, x^{j}\right), j=1, \ldots, n$ on $M$ induces a system of local coordinates $\left(\pi^{-1}(U), x^{j}, x^{\bar{j}}=p_{j}\right), \bar{j}=n+1, \ldots 2 n$ on $T^{*} M$, where $x^{\bar{j}}=p_{j}$ are the components of the covector $p$ in each cotangent space $T_{x}^{*} M$ and $x \in U$ with respect to the natural coframe $\left\{d x^{j}\right\}$. We denote the set of all tensor fields of type $(r, s)$, by $\mathfrak{J}_{S}^{r}(M), \mathfrak{I}_{S}^{r}\left(T^{*}(M)\right)$ on $M$ and $T^{*} M$ respectively.

The problem of determining infinitesimal holomorphically projective transformation on $M$ and $T M$ have been studied some authors, including (Hasegawa and Yamauchi, 1979; Hasegawa and Yamauchi, 2003; Hasegawa and Yamauchi, 2005; Tarakci et al., 2009; Gezer, 2011). Also, (Etayo and Gadea, 1992; Iscan and Magden, 2008), investigated some properties of infinitesimal paraholomorphically projective transformations on tangent bundle.

In this paper, we shall use the Levi-Civita connection of the Riemannian extension by using the horizantal and vertical lifts and we give definition and formulas almost paracomplex structure $J$. Then we research the infinitesimal paraholomorphically projective transformation on cotangent bundle with respect to the Levi-Civita connection of the Riemannian extension $\left({ }^{R} \nabla\right)$ and adapted almost paracomplex structure.

\section{MATERIAL AND METHODS}

Let $\nabla$ be an affine connection on $M$. A vector field $V$ on $M$ is called an infinitesimal projective transformation if there exist a 1-form $\Omega$ on $M$ such that

$$
\left(L_{V} \nabla\right)(X, Y)=\Omega(X) Y+\Omega(Y) X,
$$

for any $X, Y \in \mathfrak{I}_{0}^{1}(M)$, where $L_{V}$ is the Lie derivation with respect to $V$. In this case $\Omega$ is called the associated 1- form of $V$. Especially, if $\Omega=0$ then $V$ is called an infinitesimal affine transformation.

An almost paracomplex manifold is an almost product manifold $(M, J), J^{2}=I$, such that the two eigenbundles $T^{+} M$ and $T^{-} M$ associated to the two eigenvalues +1 and -1 of $J$, respectively (Cruceanu et al., 1995; Salimov et al., 2007). $(M, J)$ be an almost paracomplex manifold with affine connection $\nabla$. A vector field $V$ on $M$ is called an infinitesimal paraholomorphically projective transformation if there exist a 1-form $\Omega$ on $M$ such that

$$
\left(L_{V} \nabla\right)(X, Y)=\Omega(X) Y+\Omega(Y) X+\Omega(J X) J Y+\Omega(J Y) J X,
$$

for any $X, Y \in \mathfrak{I}_{0}^{1}(M)$. In this case $\Omega$ is also called the associated 1- form of $V$ (Prvanovic, 1971; Etayo and Gadea, 1992).

Let $X=X^{i} \frac{\partial}{\partial x^{i}}$ and $\omega=\omega_{i} d x^{i}$ be the local expressions of a vector field $X$ and a covector (1-form) field $\omega$ on $M$, respectively. According to the induced coordinates the vertical lift ${ }^{V} \omega$ of $\omega$, the horizontal lift ${ }^{H} X$ and the complete lift ${ }^{C} X$ of $X$ are obtained as follows

$$
\begin{gathered}
{ }^{V} \omega=\omega_{i} \partial_{\bar{i}}, \\
{ }^{H} X=X^{i} \partial_{i}+p_{h} \Gamma_{i j}^{h} X^{j} \partial_{\bar{i}} \\
{ }^{c} X=X^{j} \partial_{i}-p_{h} \partial_{i} X^{h} \partial_{\bar{i}},
\end{gathered}
$$


where $\partial_{i}=\frac{\partial}{\partial x^{i}}, \partial_{\bar{i}}=\frac{\partial}{\partial x^{\bar{i}}}$ and $\Gamma_{i j}^{h}$ are the coefficients of symmetric (torsion-free) affine connection $\nabla$ on $M$ (Yano and Ishihara, 1973). For arbitrary $X, Y \in \mathfrak{J}_{0}^{1}(M)$ and $\theta, \omega \in \mathfrak{I}_{1}^{0}(M)$, the Lie bracket operation of vertical and horizontal vector fields on $T^{*} M$ is given as follows

$$
\left\{\begin{array}{l}
{\left[{ }^{H} X,{ }^{H} Y\right]={ }^{H}[X, Y]+{ }^{V}(p \circ R(X, Y))} \\
{\left[{ }^{H} X,{ }^{V} \omega\right]={ }^{V}\left(\nabla_{X} \omega\right)} \\
{\left[{ }^{V} \theta,{ }^{V} \omega\right]=0,}
\end{array}\right.
$$

where $R=R(X, Y)=\left[\nabla_{X}, \nabla_{Y}\right]-\nabla_{[X, Y]}$ is the curvature tensor of the symmetric connection $\nabla$ (Yano and Ishihara, 1973).

\section{The adapted frame}

The adapted frame $\left\{E_{\alpha}\right\}=\left\{E_{j}, E_{\bar{j}}\right\}$ on each induced coordinate neighbourhood $\pi^{-1}(U)$ of $T^{*} M$ is given by (Yano and Ishihara, 1973)

$$
\left.\begin{array}{l}
E_{j}={ }^{H} X_{(j)}=\partial_{j}+p_{a} \Gamma_{h j}^{a} \partial_{\bar{h}}, \\
E_{\bar{j}}={ }^{V} \theta_{(j)}=\partial_{\bar{j}},
\end{array}\right\}
$$

where

$$
X_{(j)}=\frac{\partial}{\partial x^{j}}, \theta^{j}=d x^{j}, j=1, \ldots, n,
$$

the indices $\alpha, \beta, \gamma, \ldots=1, \ldots, 2 n$ denote the indices according to the adapted frame. It follows from (1), (2) and (4) that

$$
\begin{aligned}
& { }^{V} \omega=\left(\begin{array}{l}
0 \\
\omega_{j}
\end{array}\right) \\
& { }^{H} X=\left(\begin{array}{l}
X^{j} \\
0
\end{array}\right)
\end{aligned}
$$

according to the adapted frame $\left\{E_{\alpha}\right\}$.

Lemma 1 The Lie bracket of the adapted frame of $T^{*} M$ satisfies the following identities (Yano and Ishihara, 1973)

$$
\begin{aligned}
& {\left[E_{i}, E_{j}\right]=p_{s} R_{i j l}^{S} E_{\bar{l}},} \\
& {\left[E_{i}, E_{\bar{j}}\right]=-\Gamma_{i l}^{j} E_{\bar{l}},} \\
& {\left[E_{\bar{i}}, E_{\bar{j}}\right]=0,}
\end{aligned}
$$

where $R_{i j l}^{S}=\partial_{i} \Gamma_{j l}^{S}-\partial_{j} \Gamma_{i l}^{S}+\Gamma_{i k}^{S} \Gamma_{j l}^{k}-\Gamma_{j k}^{S} \Gamma_{i l}^{k}$ indicates the Riemannian curvature tensor of $(M, g)$.

Lemma 2 Let $V$ be a vector field of $T^{*} M$ with the components $\left(v^{h}, v^{\bar{h}}\right)$. Then, the Lie derivatives of the adapted frame and the dual basis are obtained as follows (Bilen, 2019):

1. $L_{V} E_{i}=-\left(E_{i} v^{k}\right) E_{k}-\left(v^{a} p_{s} R_{i a k}^{s}+E_{i} v^{\bar{k}}-v^{\bar{a}} \Gamma_{i k}^{a}\right) E_{\bar{k}}$,

2. $L_{V} E_{\bar{i}}=-\left(E_{\bar{i}} v^{k}\right) E_{k}-\left(v^{a} \Gamma_{a k}^{i}+E_{\bar{i}} v^{\bar{k}}\right) E_{\bar{k}}$,

3. $L_{V} d x^{h}=\left(E_{k} v^{h}\right) d x^{k}+\left(E_{\bar{k}} v^{h}\right) \delta p_{k}$,

4. $L_{V} \delta p_{h}=\left(v^{a} p_{s} R_{k a h}{ }^{s}-v^{\bar{a}} \Gamma_{k h}^{a}+\left(E_{k} v^{\bar{m}}\right) \delta_{h}^{m}\right) d x^{k}+\left(v^{a} \Gamma_{a h}^{k}+\left(E_{\bar{k}} v^{\bar{m}}\right) \delta_{h}^{m}\right) \delta p_{k}$.

\{For more work on tangent bundles see (Hasegawa and Yamauchi, 2003; Gezer, 2011)\}. 


\section{Riemannian Extension}

A pseudo-Riemannian metric ${ }^{R} \nabla \in \mathfrak{I}_{2}^{0}\left(T^{*} M\right)$ is given by (Yano and Ishihara, 1973).

$$
{ }^{R} \nabla\left({ }^{C} X,{ }^{C} Y\right)=-\gamma\left(\nabla_{X} Y+\nabla_{Y} X\right) \text {, }
$$

for any $X, Y \in \mathfrak{I}_{0}^{1}(M)$, where

$$
-\gamma\left(\nabla_{X} Y+\nabla_{Y} X\right)=p_{m}\left(X^{j} \nabla_{j} Y^{m}+Y^{j} \nabla_{j} X^{m}\right),
$$

${ }^{R} \nabla \in \mathfrak{I}_{2}^{0}\left(T^{*} M\right)$ with the following components in $\pi^{-1}(U)$

$$
{ }^{R} \nabla=\left({ }^{R} \nabla_{J I}\right)=\left(\begin{array}{cc}
-2 p_{h} \Gamma_{j i}^{h} & \delta_{j}^{i} \\
\delta_{i}^{j} & 0
\end{array}\right)
$$

relative to the natural frame, where $\delta_{j}^{i}$ is the Kronecker delta. The indices $i, j, k, \ldots=1, \ldots, 2 n$ correspond to the natural frame $\left\{\frac{\partial}{\partial x^{i}}, \frac{\partial}{\partial x^{i}}\right\}$. The analyzed tensor field defines a pseudo-Riemannian metric in $T^{*} M$ and a line element of the pseudo-Riemannian metric ${ }^{R} \nabla$ is given by the formula

$$
d s^{2}=2 d x^{i} \delta p_{i},
$$

where

$$
\delta p_{i}=d p_{i}-p_{h} \Gamma_{j i}^{h} d x^{i}
$$

This metric is called the Riemannian extension of the symmetric affine connection $\nabla$ (Patterson and Walker, 1952; Yano and Ishihara, 1973). Any tensor field of type $(0,2)$ is entirely detected by its action of ${ }^{H} X$ and ${ }^{V} \omega$ on $T^{*} M$ (Yano and Ishihara, 1973). Then the Riemannian extension ${ }^{R} \nabla$ is defined by

$$
\begin{gathered}
{ }^{R} \nabla\left({ }^{V} \omega,{ }^{V} \theta\right)=0, \\
{ }^{R} \nabla\left({ }^{V} \omega,{ }^{H} X\right)={ }^{V}(\omega(X))=(\omega(X)) \circ \pi, \\
{ }^{R} \nabla\left({ }^{H} X,{ }^{H} Y\right)=0
\end{gathered}
$$

for any $X, Y \in \mathfrak{J}_{0}^{1}(M)$ and $\omega, \theta \in \mathfrak{I}_{1}^{0}(M)$ (Aslanci et al., 2010).

\section{The Levi-Civita connection of ${ }^{R} \nabla$}

${ }^{C} \nabla$ is the Levi-Civita connection of ${ }^{R} \nabla$, because of ${ }^{C} \nabla\left({ }^{R} \nabla\right)=0$. ( ${ }^{C} \nabla$ is called the complete lift of $\nabla$ to $T^{*} M$ ) The Levi-Civita connection of ${ }^{C} \nabla$ in $\pi^{-1}(U) \subset T^{*} M$ are given by

$$
\begin{gathered}
{ }^{C} \Gamma_{j i}^{h}=\Gamma_{j i}^{h} \\
{ }^{C} \Gamma_{j i}^{\bar{h}}=-\Gamma_{j h}^{i} \\
{ }^{C} \Gamma_{j i}^{\bar{h}}=\frac{1}{2} p_{m}\left(R_{j i h}^{m}-R_{i h j}^{m}+R_{h j i}^{m}\right)=p_{m} R_{h i j}^{m} \\
{ }^{C} \Gamma_{\bar{j} i}^{h}={ }^{C} \Gamma_{j \bar{i}}^{h}={ }^{C} \Gamma_{\overline{j i}}^{h}={ }^{C} \Gamma_{\bar{j} i}^{\bar{h}}={ }^{C} \Gamma_{\bar{j} i}^{\bar{h}}=0
\end{gathered}
$$

with respect to adapted frame $\left\{E_{\alpha}\right\}$, where $\Gamma_{j i}^{h}$ denote the Christoffel symbols constructed with $g_{j i}$ on $M$ (Aslanci et al, 2010).

Let us consider a tensor field $J$ of type $(1,1)$ on $T^{*} M$ defined by

$$
J^{H} X=-{ }^{H} X, J^{V} \omega={ }^{V} \omega,
$$

for any $X \in \mathfrak{I}_{0}^{1}(M)$, i.e., $J E_{i}=-E_{i}, J E_{\bar{i}}=E_{\bar{i}}$. Then we obtain $J^{2}=I$. Therefore $J$ is an almost paracomplex structure on $T^{*} M$. This almost paracomplex structure is called adapted almost paracomplex structure (Etayo and Gadea, 1992). 


\section{RESULTS AND DISCUSSION}

Theorem 3 Let $(M, g)$ be a Riemannian manifold and $T^{*} M$ be its cotangent bundle with the Riemannian extension and adapted almost paracomplex structure. A vector field $V$ is an infinitesimal paraholomorphically projective transformation with associated 1 - form $\Omega$ on $T^{*} M$ if and only if there exist $B=\left(B^{h}\right) \in \mathfrak{I}_{0}^{1}(M), D=\left(D_{h}\right) \in \mathfrak{I}_{1}^{0}(M)$ and $A=\left(A_{i}^{h}\right), C=\left(C_{i}^{h}\right) \in \mathfrak{I}_{1}^{1}(M)$ satisfying

1. $\left(\begin{array}{c}v^{k} \\ v^{k}\end{array}\right)=\left(\begin{array}{c}p^{s} A_{s}^{k}+B^{k} \\ D_{k}+p_{a} C_{k}^{a}+4 \varphi p_{k}+2 p_{a} p_{k} \Psi^{a}\end{array}\right)$

2. $\nabla_{j} A^{k i}=0, \nabla_{j} C_{i}^{i}=0$

3. $\nabla_{j} \varphi=0, \nabla_{j} \psi=0, \nabla_{j} \Psi^{i}=0$

4. $A^{i a} R_{a i j}^{S}=0$

5. $A_{s}^{a} R_{a i j}^{k}+A_{h}^{k} R_{s i j}^{h}=0$

6. $\nabla_{i} \nabla_{j} B^{k}+B^{a} R_{a i j}^{k}=2 \Omega_{i} \delta_{j}^{k}+2 \Omega_{j} \delta_{i}^{k}=L_{B} \Gamma_{i j}^{k}$

7. $\nabla_{i} R_{j a k}^{S}-\nabla_{a} R_{k j i}^{S}=0$

8. $R_{j h i}^{S} \Psi^{h}=0$

9. $\nabla_{i} \nabla_{j} D_{k}+D_{a} R_{k j i}^{a}=0$

10. $C_{k}^{h} R_{j i h}^{s}+C_{a}^{s} R_{i j k}^{a}=0$

11. $\Omega_{j}=\frac{1}{4 n} \nabla_{i} \nabla_{j} B^{j}, \Omega_{\bar{j}}=\Psi^{j}$

where $V=\left(\begin{array}{l}v^{k} \\ v^{\bar{k}}\end{array}\right)=v^{k} E_{k}+v^{\bar{k}} E_{\bar{k}}, \Omega=\left(\Omega_{j} d x^{j}+\Omega_{\bar{j}} \delta y^{j}\right)$.

Proof. Here we prove only the necessary condition because it is easy to prove the sufficient condition. Let $V$ be an infinitesimal paraholomorphically projective transformation with the associated 1- form $\Omega$ on $T^{*} M$

for any $X, Y \in \mathfrak{J}_{0}^{1}(M)$.

$$
\left(L_{V} \nabla\right)(X, Y)=\Omega(X) Y+\Omega(Y) X+\Omega(J X) J Y+\Omega(J Y) J X
$$

From

we obtain

$$
\left(L_{V}{ }^{R} \nabla\right)\left(E_{\bar{i}}, E_{\bar{j}}\right)=\Omega\left(E_{\bar{i}}\right) E_{\bar{j}}+\Omega\left(E_{\bar{j}}\right) E_{\bar{i}}+\Omega\left(J E_{\bar{i}}\right) J E_{\bar{j}}+\Omega\left(J E_{\bar{j}}\right) J E_{\bar{i}}
$$

$$
\left(L_{V}{ }^{R} \nabla\right)\left(E_{\bar{i}}, E_{\bar{j}}\right)=2\left(\Omega_{\bar{i}} \delta_{k}^{j}+\Omega_{\bar{j}} \delta_{k}^{i}\right) E_{\bar{k}}
$$

also

$$
\left(L_{V}{ }^{R} \nabla\right)\left(E_{\bar{i}}, E_{\bar{j}}\right)=\left[\partial_{\bar{i}}\left(\partial_{\bar{j}} v^{k}\right)\right] E_{k}+\left[\partial_{\bar{i}}\left(\partial_{\bar{j}} v^{\bar{k}}\right)\right] E_{\bar{k}}
$$

from (5) and (6) we obtain

$$
\partial_{\bar{i}}\left(\partial_{\bar{j}} v^{k}\right)=0 \Rightarrow v^{k}=p^{s} A_{s}^{k}+B^{k}
$$

and

$$
\partial_{\bar{i}}\left(\partial_{\bar{j}} v^{\bar{k}}\right)=2\left(\Omega_{\bar{i}} \delta_{k}^{j}+\Omega_{\bar{j}} \delta_{k}^{i}\right) .
$$

Contracting $k$ and $j$ in (8), we have

$$
\Omega_{\bar{i}}=\partial_{\bar{i}} \psi,
$$


where $\psi=\frac{1}{2 n+2} \partial \bar{j} v^{\bar{j}}$. If we use the expression (9) in (8), expression (8) is rewritten as follows:

$$
\partial_{\bar{i}}\left(\partial_{\bar{j}} v^{\bar{k}}\right)=2\left(\partial_{\bar{i}} \psi\right) \delta_{k}^{j}+2\left(\partial_{\bar{j}} \psi\right) \delta_{k}^{i} .
$$

Differentiating (10) partially, we have

$$
\begin{aligned}
\partial_{\bar{h}} \partial_{\bar{i}} \partial_{\bar{j}} v^{\bar{k}} & =2 \partial_{\bar{h}} \partial_{\bar{i}} \psi \delta_{k}^{j}+2 \partial_{\bar{h}} \partial_{\bar{j}} \psi \delta_{k}^{i} \\
& =2 \partial_{\bar{h}} \partial_{\bar{i}} \psi \delta_{k}^{j}+2 \partial_{\bar{h}} \partial_{\bar{i}} \psi \delta_{k}^{i} \delta_{i}^{j} \\
& =\partial_{\bar{h}} \partial_{\bar{i}}\left(4 \psi \delta_{k}^{j}\right)
\end{aligned}
$$

from here we get

$$
\partial_{\bar{h}} \partial_{\bar{i}}\left(\partial_{\bar{j}} v^{\bar{k}}-4 \psi \delta_{k}^{j}\right)=0
$$

Written here as

$$
M_{k}^{i j}=\partial_{\bar{i}}\left(\partial_{\bar{j}} v^{\bar{k}}-4 \psi \delta_{k}^{j}\right)
$$

and

$$
C_{k}^{j}+p_{a} M_{k}^{a j}=\partial_{\bar{j}} v^{\bar{k}}-4 \psi \delta_{k}^{j}
$$

where $C_{k}^{j}$ and $M_{k}^{i j}$ are certain functions which depend only on the variables $\left(x^{h}\right)$. Also

$$
M_{k}^{i j}+M_{k}^{j i}=\partial_{\bar{i}} \partial_{\bar{j}} v^{\bar{k}}-4 \partial_{\bar{i}} \psi \delta_{k}^{j}+\partial_{\bar{j}} \partial_{\bar{i}} v^{\bar{k}}-4 \partial_{\bar{j}} \psi \delta_{k}^{i} .
$$

Using (10) in above equation

$$
M_{k}^{i j}=\frac{1}{2}\left(M_{k}^{i j}-M_{k}^{j i}\right)=2\left[\left(\partial_{\bar{j}} \psi\right) \delta_{k}^{i}-\left(\partial_{\bar{i}} \psi\right) \delta_{k}^{j}\right] .
$$

Contracting $k$ and $j$ in (12), we have

$$
C_{k}^{k}+p_{a} M_{k}^{a k}=(2-2 n) \psi .
$$

From which

$$
\psi=\frac{1}{2-2 n} C_{k}^{k}+p_{a} \frac{1}{2-2 n} M_{k}^{a k}
$$

and we get

$$
\psi=\varphi+p_{a} \Psi^{a},
$$

where $\varphi=\frac{1}{2-2 n} C_{k}^{k}$ and $\Psi^{a}=\frac{1}{2-2 n} M_{k}^{a k}$, from which we have

$$
\Omega_{\bar{i}}=\partial_{\bar{i}} \psi=\Psi^{i} \text {. }
$$

If used (13) and (14) in (12) we get

$$
\partial_{\bar{j}} v^{\bar{k}}=C_{k}^{j}+4 \varphi \delta_{k}^{j}+2 p_{a} \Psi^{a} \delta_{k}^{j}+2 p_{k} \Psi^{j}
$$

and

$$
v^{\bar{k}}=D_{k}+p_{a} C_{k}^{a}+4 \varphi p_{k}+2 p_{a} p_{k} \Psi^{a},
$$

where $D_{k}$ are certain functions which depend only on $\left(x^{h}\right)$. The coordinat transformation rule implies that $D=\left(D_{k}\right) \in \mathfrak{I}_{1}^{0}(M)$.

Next, from

$$
\left(L_{V} \nabla\right)(X, Y)=\Omega(X) Y+\Omega(Y) X+\Omega(J X) J Y+\Omega(J Y) J X
$$

we have

$$
\left(L_{V}^{R} \nabla\right)\left(E_{\bar{i}}, E_{j}\right)=0
$$

or 


$$
\left(L_{V}^{R} \nabla\right)\left(E_{i}, E_{\bar{j}}\right)=0
$$

from which, we get

$$
0=\left[\nabla_{j} A^{k i}\right] E_{k}+\left[A^{i a} p_{s} R_{k a j}^{s}+v^{a} R_{j a k}^{i}+\nabla_{j} C_{k}^{i}+2 p_{k}\left(\nabla_{j} \Psi^{i}\right)+2 p_{a} \delta_{k}^{i} \nabla_{j} \Psi^{a}+4\left(\partial_{j} \varphi\right) \delta_{k}^{i}\right] E_{\bar{k}} .
$$

Therefore,

$$
\nabla_{j} A^{k i}=0
$$

and

$$
A^{i a} p_{s} R_{k a j}^{s}+v^{a} R_{j a k}^{i}+\nabla_{j} C_{k}^{i}+2 p_{s}\left(\nabla_{j} \Psi^{s}\right)+2 p_{a} \delta_{k}^{i} \nabla_{j} \Psi^{a}+4\left(\partial_{j} \varphi\right) \delta_{k}^{i}=0 .
$$

Contracting $k$ and $i$ in (18), we have

$$
\left\{\begin{array}{l}
\nabla_{j} C_{i}^{i}=0 \\
\nabla_{j} \varphi=0
\end{array}\right.
$$

and

$$
\nabla_{j} \Psi^{s}=\frac{1}{2(n+1)} A^{i a} R_{a i j}^{s}
$$

Lastly, from

$$
\left(L_{V}^{R} \nabla\right)\left(E_{i}, E_{j}\right)=\left(2 \Omega_{i} \delta_{j}^{k}+2 \Omega_{j} \delta_{i}^{k}\right) E_{k}
$$

we obtain

$$
\begin{aligned}
\left(2 \Omega_{i} \delta_{j}^{k}+2 \Omega_{j} \delta_{i}^{k}\right) E_{k}=\left[\nabla_{i} \nabla_{j} v^{k}+v^{a} R_{a i j}^{k}+A^{h k} p_{s} R_{h j i}^{s}\right] E_{k} \\
+\left[p_{s}\left(\left(\nabla_{i} v^{h}\right) R_{k h j}^{s}+\left(\nabla_{j} v^{h}\right) R_{k h i}^{s}-\left(E_{\bar{h}} v^{\bar{k}}\right) R_{h j i}^{S}\right)\right. \\
\left.+v^{a} p_{s}\left(\nabla_{i} R_{j a k}^{s}-\nabla_{a} R_{k j i}^{s}\right)+\left(v^{\bar{a}} R_{k j i}^{a}+\nabla_{i} \nabla_{j} v^{\bar{k}}\right)\right] E_{\bar{k}}
\end{aligned}
$$

from which, using (7) and (16), we obtain

$$
\begin{gathered}
\nabla_{i} \nabla_{j} A_{s}^{k}+A_{s}^{a} R_{a i j}^{k}+A_{h}^{k} R_{s i j}^{h}=0, \\
\nabla_{i} \nabla_{j} B^{k}+B^{a} R_{a i j}^{k}=2 \Omega_{i} \delta_{j}^{k}+2 \Omega_{j} \delta_{i}^{k}=L_{B} \Gamma_{i j}^{k}, \\
\nabla_{i} R_{j a k}^{s}-\nabla_{a} R_{k j i}^{s}=0, \\
\nabla_{i} \nabla_{j} \Psi^{s}+R_{j h i}^{s} \Psi^{h}=0, \\
\nabla_{i} \nabla_{j} D_{k}+D_{a} R_{k j i}^{a}=0, \\
\left(\nabla_{i} B^{a}\right) R_{k a j}^{s}+\left(\nabla_{j} B^{a}\right) R_{k a i}^{s}+C_{k}^{h} R_{j h i}^{s}+C_{a}^{s} R_{k j i}^{a}+\nabla_{i} \nabla_{j} C_{k}^{s}=0 .
\end{gathered}
$$

From (26), we get

$$
\begin{gathered}
K_{i j}=\left(\nabla_{i} B^{a}\right) R_{k a j}^{s}+\left(\nabla_{j} B^{a}\right) R_{k a i}^{s}+C_{k}^{h} R_{j h i}^{s}+C_{a}^{s} R_{k j i}^{a}+\nabla_{i} \nabla_{j} C_{k}^{s}=0, \\
K_{j i}=\left(\nabla_{j} B^{a}\right) R_{k a i}^{s}+\left(\nabla_{i} B^{a}\right) R_{k a j}^{s}+C_{k}^{h} R_{i h j}^{s}+C_{a}^{s} R_{k i j}^{a}+\nabla_{j} \nabla_{i} C_{k}^{s}=0 . \\
K_{i j}-K_{j i}=C_{k}^{h}\left(R_{j h i}^{s}-R_{i h j}^{s}\right)+C_{a}^{s}\left(R_{k j i}^{a}-R_{k i j}^{a}\right)=0 \\
C_{k}^{h} R_{j i h}^{s}+C_{a}^{s} R_{i j k}^{a}=0
\end{gathered}
$$

Contracting $j$ and $k$ in (22), we obtain

$$
\Omega_{i}=\frac{1}{4 n} \nabla_{i} \nabla_{j} B^{j}
$$

This completes the proof.

Theorem 4 Let $(M, g)$ be a Riemannian manifold and $T^{*} M$ be its cotangent bundle with the Riemannian 
extension and adapted almost paracomplex structure. If $T^{*} M$ admits a non-affine infinitesimal paraholomorphically projective transformation, than $M$ and $T^{*} M$ are locally flat.

Proof. Let $V$ be non-affine infinitesimal paraholomorphically projective transformation on $T^{*} M$, using (3) in the expression of theorem 3, we have $\nabla_{i}\|\Psi\|^{2}=\nabla_{j}\|\partial \psi\|^{2}=0$. Hence, $\|\Psi\|$ and $\|\partial \psi\|$ are constant on $M$. Suppose that $M$ is non-locally flat, then $\Psi=\partial \psi=0$ by virtue of (9) and (3) in the expression of theorem 3, that is, $V$ is an infinitesimal affine transformation. This is a contradiction. Therefore, $M$ is locally flat. In this case $T^{*} M$ is locally flat.

Corollary 5 Let $(M, g)$ be a Riemannian manifold and $T^{*} M$ be its cotangent bundle with the Riemannian extension and adapted almost paracomplex structure. A vertical vector field $V$ is an infinitesimal paraholomorphically projective transformation with associated 1 - form $\Omega$ on $T^{*} M$ if and only if there exist $D=\left(D_{h}\right) \in \mathfrak{I}_{1}^{0}(M)$ and $C=\left(C_{i}^{h}\right) \in \mathfrak{I}_{1}^{1}(M)$ satisfying

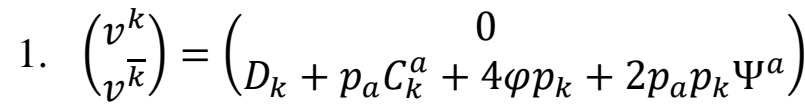

2. $\nabla_{j} C_{k}^{i}=0$

3. $\nabla_{j} \varphi=0, \nabla_{j} \psi=0, \nabla_{j} \Psi^{i}=0$

4. $\nabla_{i} \nabla_{j} D_{k}+D_{a} R_{k j i}^{a}=0$

5. $C_{a}^{s} R_{k j i}^{a}+C_{k}^{h} R_{j h i}^{s}=0$

6. $\Psi^{h} R_{j h i}^{s}=0$

7. $\Psi^{a} R_{j k i}^{s}+\Psi^{s} R_{k j i}^{a}=0$

8. $\Omega_{j}=0, \Omega_{\bar{j}}=\Psi^{j}$

where $V=\left(\begin{array}{l}0 \\ v^{\bar{k}}\end{array}\right)=v^{\bar{k}} E_{\bar{k}}, \Omega=\left(\Omega_{j} d x^{j}+\Omega_{\bar{j}} \delta y^{j}\right)$.

\section{CONCLUSION}

In this article, we use the Levi-Civita connection of the Riemannian extension and we give definition and formulas almost paracomplex structure $J$. Then we research the infinitesimal paraholomorphically projective transformation on cotangent bundle with respect to the Levi-Civita connection of the Riemannian extension $\left({ }^{R} \nabla\right)$ and adapted almost paracomplex structure $J$.

\section{REFERENCES}

Aslanci S, Kazimova S and Salimov A A, 2010. Some remarks concerning Riemannian extensions. Ukrainian Mathematical Journal, 62(5): 661-675.

Bilen L, 2019. Projective Vector Fields on the Cotangent Bundle with Modified Riemannian Extension. Journal of the Institute of Science and Technology, 9(1): 389-396.

Cruceanu V, Gadea PM and Munoz Masque J, 1995. Para-Hermitian and Para-Kahler Manifolds. Quaderni Inst. Math. Messina, 2: 1-70.

Etayo F and Gadea PM, 1992. Paraholomorphically projective vector field. An. St.Univ."Al. I. Cuza" Iaşi Sect. a Mat. (N. S.), 38: 201-210.

Gezer A, 2011. On infinitesimal holomorfically projective transformations on the tangent bundles with 
respect to the Sasaki metric. Proc. Est. Acad. Sci, 60(3): 149-157

Hasegawa I and Yamauchi K, 1979. On infinitesimal holomorphically projective transformations in compact Kaehlerian manifolds. Hokkaido Math. J, 8: 214-219.

Hasegawa I and Yamauchi K, 2003. Infinitesimal holomorphically projective transformations on the tangent bundles with horizontal lift connection and adapted almost complex structure. J. Hokkaido Univ. Education, 53: 1-8.

Hasegawa I and Yamauchi K, 2005. Infinitesimal holomorphically projective transformations on the tangent bundles with complete lift connection. Differ. Geom. Dyn. Syst, 7: 42-48.

Iscan $\mathrm{M}$ and Magden A, 2008. Infinitesimal paraholomorphically projective transformations on tangent bundles with diagonal lift connection. Differential Geometry - Dynamical Systems, Vol.10: 170-177.

Patterson E M and Walker A G, 1952. Riemannian extensions. Quant. J. Math, 3: 19-28.

Prvanovic M, 1971. Holomorphically projective transformations in a locally product spaces. Math. Balkanika (N.S.), 1: 195-213.

Salimov A A, Iscan M and Etayo F, 2007. Paraholomorphic B-manifold and its properties. Topology and its Application, 154: 925-933.

Tarakci O, Gezer A and Salimov A A, 2009. On solutions of IHPT equations on tangent bundle with the metric II+III. Math.Comput. Modelling, 50: 953-958.

Yano K, Ishihara S, 1973. Tangent and cotangent bundles. Marcel Dekker, Inc. New York. 